\title{
Crystallographically-Oriented Nanoassembly by ZnS Tricrystals and Subsequent Three-Dimensional Epitaxy
}

\author{
Yingchun Zhu ( $\varangle)$, Qichao Ruan, and Fangfang Xu
}

The Key Laboratory of Inorganic Coating Materials, Shanghai Institute of Ceramics, Chinese Academy of Sciences, Shanghai 200050, China

Received: 19 April 2009 / Revised: 19 May 2009 / Accepted: 8 July 2009

CTsinghua University Press and Springer-Verlag 2009. This article is published with open access at Springerlink.com

\begin{abstract}
Chemistry gives us the ability to manipulate atoms and molecules into nanometer and micrometer scale building blocks, while the science of crystallography is concerned with the spatial arrangement of atoms, ions, and molecules and thus the morphology and structures of materials. Complex three-dimensional ZnS nanostructures have been fabricated via step-by-step crystallographically-controlled chemical processes. Tricrystals of ZnS whiskers were prepared via a controlled thermal evaporation process, and then the tricrystals were thermally treated in an atmosphere formed by evaporating B-N-O precursors into $\mathrm{N}_{2} / \mathrm{NH}_{3}$ to afford $\mathrm{BN}$ coated arrays of nanobranches. The ZnS nanobranches grew epitaxially on the ternary facets and extended in three [0001] directions forming ordered nanostructures. Meanwhile, the protecting insulating sheath of BN formed on the $\mathrm{ZnS}$ nanostructures confined the growth of the nanospines and enhanced their stability. The method may be extended to fabricate other semiconductor nanomaterials with novel structures.
\end{abstract}

\section{KEYWORDS}

ZnS tricrystal, crystallograpically-controlled chemical process, nanoassembly, three-dimensional epitaxy

\section{Introduction}

Chemistry gives us the ability to manipulate atoms and molecules into nanometer and micrometer scale building blocks which can serve as tailored functional materials. The chemical approach has achieved considerable progress in assembling nanostructures and nanofabrication [1-7]. In contrast to physical processes based on lithography techniques $[8,9]$, chemistry-based self-assembly is a low cost and high yield approach for the preparation of nanostructures, and is expected to result in more and more nanostructures with novel physical and chemical properties.

The science of crystallography is concerned with the spatial arrangement of atoms, ions, and molecules in crystalline states and thus the morphology and structures of materials. As expected, more and more studies have revealed that the growth of nanostructures is generally based on the crystallographic characteristics of the materials, and that this growth can be kinetically controlled along various crystallographic directions to afford various shapes of nanostructures [10-14]. For example,

Address correspondence to yingchunzhu@yahoo.com 
wurtzite phase materials tend to grow along the [0001] direction [10-14] and zinc blende phase materials tend to grow along the [111] direction [13 -16]. Motivated by this principle, we report here further investigations of the fabrication of complex nanostructures on the basis of crystallography via chemical processes.

$\mathrm{ZnS}$ is an important II-IV semiconductor, with representative crystal polymorphs, which is widely used in ultraviolet light-emitting diodes and lasers and other devices [15-20]. There has been considerable progress in the synthesis of $\mathrm{ZnS}$ nanostructures such as nanoparticles, nanowires, nanobelts, nanotubes, nanocables, and nanotetrapods $[13,14,21-31]$. Here we report a chemical route to fabricate complex three-dimensional $\mathrm{ZnS}$ nanostructures. The method may be extended to fabricate other semiconductor nanomaterials and other novel nanostructures may be obtained via such controlled chemical process.

\section{Experimental}

ZnS tricrystal whiskers were prepared by evaporating ZnS powder at $1250{ }^{\circ} \mathrm{C}$ under a $\mathrm{N}_{2}$ flow using a horizontal electrical resistance furnace. A graphite boat containing $\mathrm{ZnS}$ powder was inserted into a graphite tube. All the components were enclosed into a quartz tube. ZnS tricrystal whiskers were collected on the wall of the graphite tube at the downstream end, where the deposition temperatures was $\sim 400{ }^{\circ} \mathrm{C}$. Complex ZnS nanostructures were prepared by using $\mathrm{ZnS}$ tricrystal whiskers as precursors in an radio frequency (RF) induction furnace. A homemade $\mathrm{B}-\mathrm{N}-\mathrm{O}$ precursor was used to coat $\mathrm{BN}$ sheathes on $\mathrm{ZnS}$ nanostructures $[32,33]$. The $\mathrm{B}-\mathrm{N}-\mathrm{O}$ powder was put into a graphite crucible, above which a BN disc with ZnS tricrystal whiskers was arranged. All the components were enclosed into a graphite susceptor which was held in a flowing $\mathrm{N}_{2}$ atmosphere $(1.5 \mathrm{~L} / \mathrm{min})$. An $\mathrm{RF}$ induction furnace was used for heating the susceptor. The temperature of the susceptor was controlled to ensure that the $\mathrm{B}-\mathrm{N}-\mathrm{O}$ precursor was heated to 1700 ${ }^{\circ} \mathrm{C}$, whereas the $\mathrm{ZnS}$ tricrystal whiskers were heated to $900{ }^{\circ} \mathrm{C}$. A $\mathrm{N}_{2} / \mathrm{NH}_{3}$ flow $\left(\mathrm{N}_{2} 1.5 \mathrm{~L} / \mathrm{min}, \mathrm{NH}_{3} 0.05\right.$ $\mathrm{L} / \mathrm{min}$ ) was passed through the $\mathrm{B}-\mathrm{N}-\mathrm{O}$ precursor and then the ZnS twin crystals. After $1.5 \mathrm{~h}$, BN sheathed $\mathrm{ZnS}$ nanostructures were obtained. The as-prepared product was characterized by a field emission scanning electron microscopy (SEM, JSM-6700F) and field emission high-resolution transmission electron microscopy (HRTEM, JEM-2100F) operated at $200 \mathrm{kV}$.

\section{Results and discussion}

Figure 1 shows the SEM morphology of the ZnS tricrystal microwhiskers. Rectangular ridges and triangular grooves can be seen in the center of the whiskers in Fig. 1(a), as labeled with " $\mathrm{M}$ " and "N" respectively. Each rectangular ridge represents a single crystalline part of the tricrystal, while each triangular groove is made of two neighboring parts. The cross-section image of the whiskers (Fig. 1(b)) clearly reveals that the whiskers are ternary crystals. One part of the ternary crystal acts as the "core" forming twins with other two crystals using its

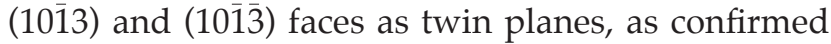
by SAED and HRTEM analysis (Fig. 1(d) and the following discussion).

Figure 1(c) shows the TEM image of a ZnS tricrystal. The image of the tricrystal shows apparent black-white contrast due to the orientations of the three parts of the ternary crystal. The dark center part corresponds to a part stretching out of the paper surface, while the bright side parts correspond to the profiles of the other two crystals. It is difficult to obtain an HRTEM image of the interface of the bright side parts at the center of the tricrystal whisker because of the thickness of the dark center part in Fig. 1(c). Figure 1(d) displays the HRTEM image of an interface taken at the end of the tilted tricrystal whisker. The image reveals two sets of (0111) lattice fringes and the interface between them. The (011) $)$ planes make an angle of ca. $80^{\circ}$ with the (1013) interface. Inset in Fig. 1(d) is the corresponding SAED pattern of the image projected along the [7523] axis, which consists of two sets of spots with a common (1013) reciprocal vector, indicating a twinrelated configuration with the twin plane on (1013). The reflection spots correspond to the bright side parts of the tricrystal.

Figure 2 displays SEM images of the ZnS 


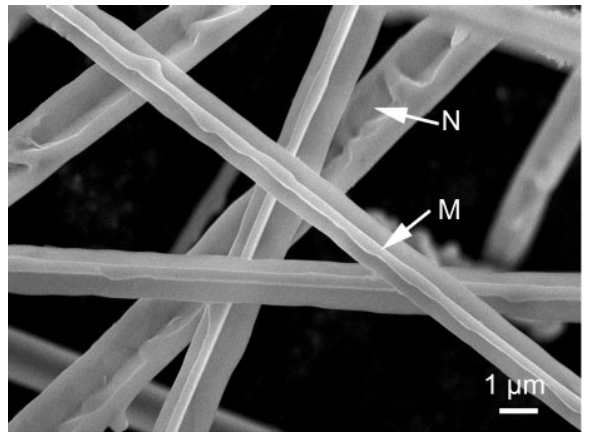

(a)

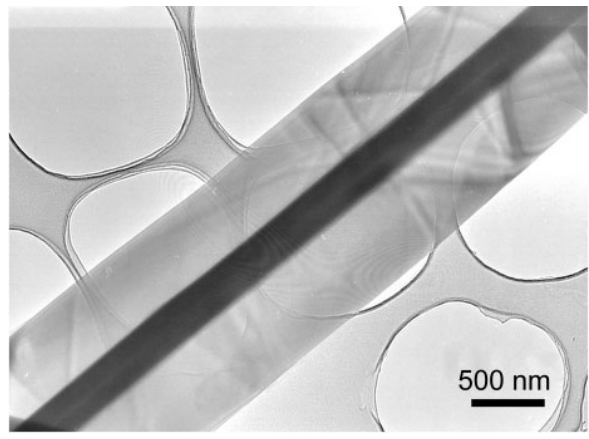

(c)

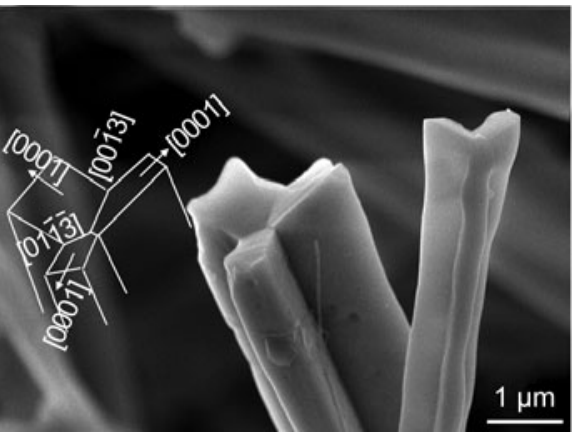

(b)

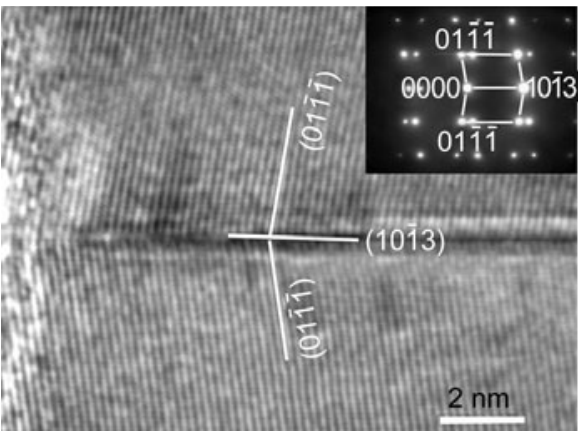

(d)

Figure 1 Morphologies and structures of ZnS tricrystals: (a) SEM image of ZnS tricrystal whiskers; (b) cross-section of ZnS tricrystal whiskers; (c) TEM image of ZnS tricrystal whiskers; (d) HRTEM image taken from the end of the tilted tricrystal whisker. Inset: SAED pattern of the tricrystal recorded along the [7523] zone axis, corresponding to the HRTEM image in (d)

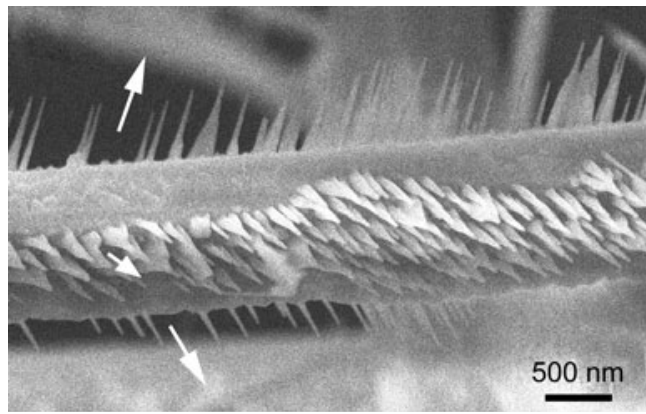

(a)

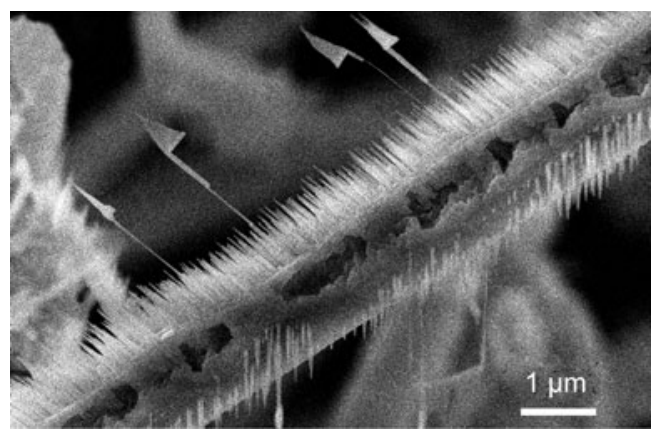

(b)

Figure 2 SEM images of nanostructures grown from the ZnS tricrystal via three-dimensional epitaxy: (a) arrays of nanospines viewed from the ridge side of the tricrystal; (b) arrays of nanospines viewed from the groove of the tricrystal nanostructures grown from tricrystal whiskers. Figure 2(a) shows arrays of nanospines which are aligned on the three sides of a tricrystal whisker viewed from the ridge. Figure 2(b) shows an image of the nanoarchitectures viewed from the groove of the tricrystal indicated in Fig. 1(a). Several "triangular flags" can be observed stretching out of the tricrystal, which have straight masts as long as about $3 \mu \mathrm{m}$. The axis direction of the masts is consistent with that of the arrays of nanospines.

The arrays of $\mathrm{ZnS}$ nanospines were further characterized by TEM and HRTEM, as shown in Fig. 3. Figure 3(a) displays a typical structure of ZnS nanospine arrays grown on ZnS tricrystal whiskers. These nanospines have sharp tips and wide bottoms (ca. $100 \mathrm{~nm}$ wide) at the joint with the whiskers. The nanospines grow in the same orientation, as revealed in the defocused SAED pattern in Fig. 3(b). Each nanospine is a single crystal as evidenced by the SAED pattern (inset in Fig. 3(c)). The SAED pattern can be indexed as a wurtzite phase with the lattice constants $a=0.382 \mathrm{~nm}$ and $c=0.625 \mathrm{~nm}$ 


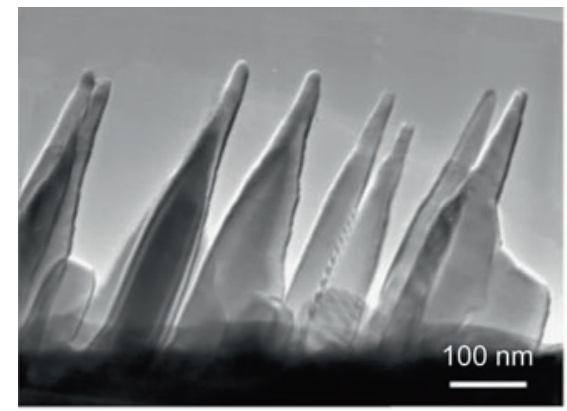

(a)

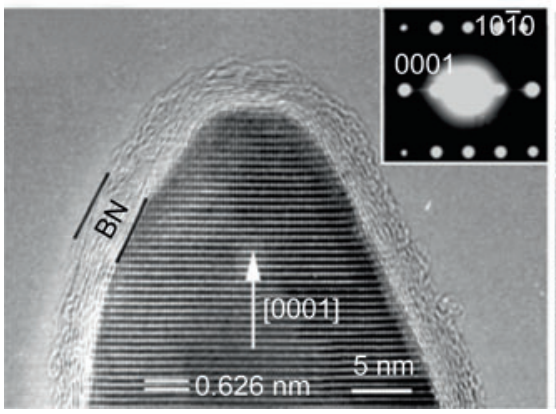

(c)

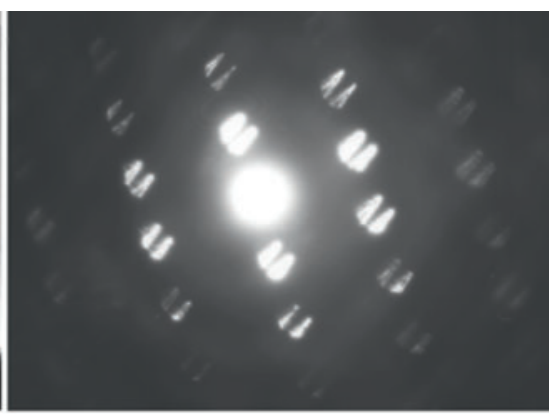

(b)

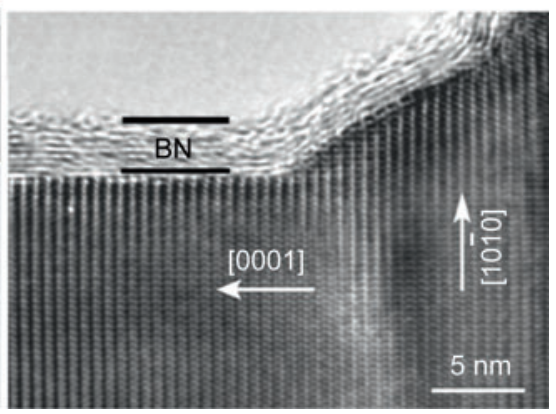

(d)

Figure 3 TEM images of BN-sheathed ZnS nanostructures: (a) arrays of nanospines; (b) defocused SAED pattern of the nanospine arrays; (c) HRTEM image of the tip of a nanospine: a single crystal nanospine coated with a uniform BN sheath. Inset: SAED pattern of the nanospines recorded along the $[\overline{1} 2 \overline{1} 0]$ zone axis. (d) Cross-section of the nanospine and trunk

(JCPDS Card: 36-1450) recorded along the [1210] zone axis. Figure 3(c) is the HRTEM image of the tip of a $\mathrm{ZnS}$ nanospine clearly revealing the lattice with the interplanar spacing $d_{001}=6.26 \AA$, and confirming [0001] as the preferred growth direction for the $\mathrm{ZnS}$ nanospines. In particular, it clearly reveals that layered $\mathrm{BN}$ sheaths are uniformly coated on the nanospines. Figure 3(d) depicts the area of the joint between a nanospine and its whisker trunk, showing a single crystal structure without an interface between the nanospine and the trunk.

We have found that the whiskers are tricrystals with twin planes of $\{1013\}$, and the arrays of nanobranches stretch out from three parts of the tricrystals along three [0001] directions. Based on the above results, a crystallographic model of the ZnS tricrystals can be proposed as schematically illustrated in Fig. 4. The crystal part $(\alpha)$ shares the twin plane of (1013) with the crystal part $(\gamma)$, and shares the twin plane of $(01 \overline{1} \overline{3})$ with the crystal part $(\beta)$. The three parts of the tricrystals share the zone axis of [3301]]. That is to say, the tricrystal whiskers

grow along the [3$\overline{3} 0 \overline{1}]$ direction with the three interfaces, i.e., (1013) and $(01 \overline{1} \overline{3})$ twin planes and a twin boundary, as illustrated in Fig. 1(b). The crystal parts $(\beta)$ and $(\gamma)$ may make an interface which is parallel to the (1120) planes of the crystal part $(\alpha)$. The $(10 \overline{1} 3)$ and $(01 \overline{1} \overline{3})$ planes make an angle of ca. $125^{\circ}$, while the (1013) and $(01 \overline{1} \overline{3})$ planes make angles of $117.5^{\circ}$ with the (112̄0) plane. The twins with $\{1013\}$ type boundary show atomic-scale twinning and should be of low energy, while the twin boundary between parts $(\beta)$ and $(\gamma)$ does not show such twinning. We found two different interfaces between $\mathrm{ZnS}$ nanospine arrays after the reheating treatment of the tricrystals, i. e., the hollow groove one (Fig. 2(b) and the compact one (Fig. 5), which may correspond to the different twin boundaries shown in Fig. 4. It was found that the wurtzite bicrystal whiskers also have twin boundaries of $\{1013\}$ planes [33]. However, the growth direction of bicrystal whiskers is different from that of the tricrystal wurtzite whiskers. The bicrystals grow along the direction (3032), while tricrystals may grow along the direction [ $3 \overline{3} 0 \overline{1}]$.

Wurtzite phase materials have been synthesized as branched structures, including nanosaws, nanocombs, and nanocantilevers [10, 11]. All the branches of these nanostructures grow along [0001], as the nanospines grow on the tricrystal whiskers. The preferential orientation of the wurtzite family is consistent with their crystallographic characteristics. The formation of branch arrays can be explained in the frame of the crystal chemical bond method [34]. The crystal structure of wurtzite $\mathrm{ZnS}$ may be interpreted as stacked $\left\{\mathrm{ZnS}_{4}\right\}$ tetrahedra sharing their common corners along the $c$ axis. The growth direction of the $\mathrm{ZnS}$ crystal is determined by the relative stacking rate of the constituent tetrahedra on various crystal faces, and the stacking rate is strongly dependent on the bonding force between atoms in 


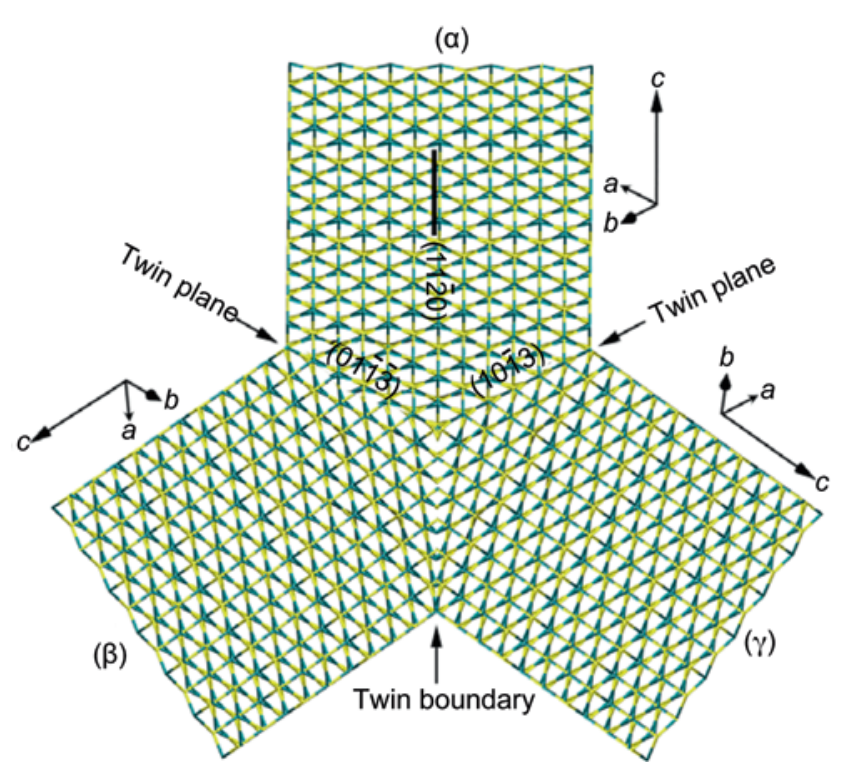

Figure 4 Crystallographic model for the ZnS tricrystals viewed along the $[3 \overline{3} 0 \overline{1}]$ zone axis

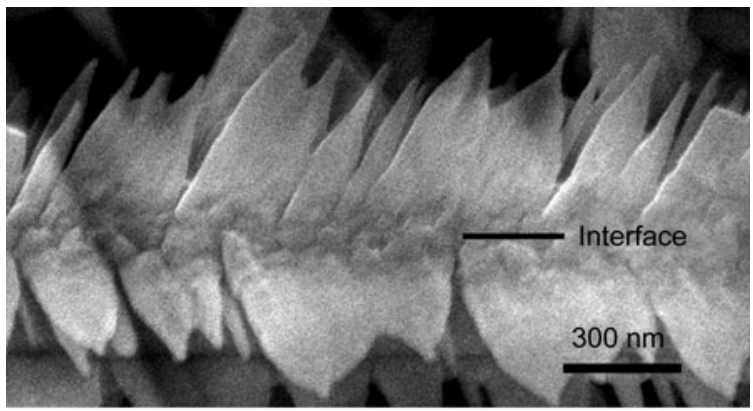

(a)

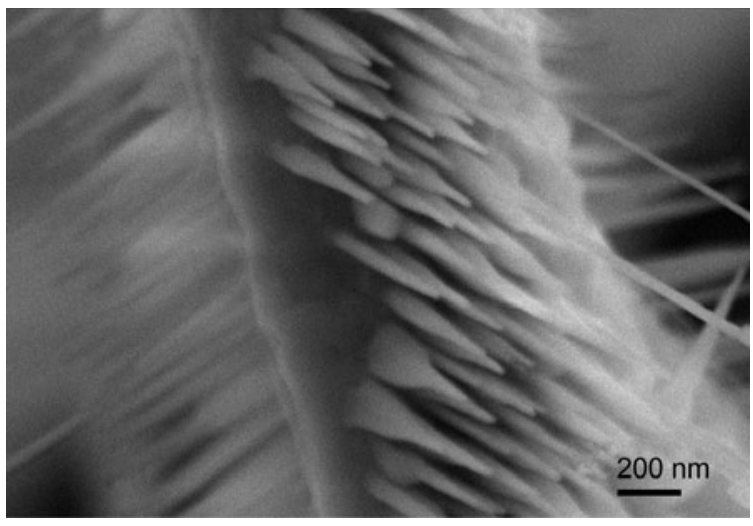

(b)

Figure 5 SEM images of interface between the nanospine arrays grown from a ZnS tricrystal

the tetrahedra at the interface. The atom at the corner of a tetrahedron has a stronger bonding force than the atoms at other positions, thus crystals grow fast along the directions where the tetrahedron corners point. Each tetrahedron has corners in the [0001] direction, which favors the growth of $\mathrm{ZnS}$ nanocrystals along the [0001] axis [13, 14]. The wurtzite family can also be described as a number of alternating planes composed of four-coordinate cations and anions. The cation and anion tetrahedra alternatively stack along the $c$ axis. The oppositely charged ions produce positively charged (0001)-cation and negatively charged (0001)-anion polar surfaces. The polarity of the wurtzite structure induces a unique asymmetric growth on the two opposite surfaces $[8,9]$. The positively charged (0001)-cation surface may provide a self-catalyzed process for the growth of nanoteeth arrays, as observed in the cases of $\mathrm{ZnO}, \mathrm{CdSe}, \mathrm{ZnSe}$, and ZnS [10, 11, 23-25, 30, 31].

A BN sheath was coated onto $\mathrm{ZnS}$ arrays of nanospines in the formation process through evaporating $\mathrm{B}-\mathrm{N}-\mathrm{O}$ precursors into a $\mathrm{N}_{2} / \mathrm{NH}_{3}$ atmosphere. The BN sheath may play a role in the formation of the nanospines. From the interface between $\mathrm{BN}$ and $\mathrm{ZnS}$, it is observed that the $\mathrm{BN}$ layers with (0001) facets are closely bound to the (1011) facets of ZnS with periodic lattice connections (Fig. 6). The coherence relationship between the BN sheath and the $\mathrm{ZnS}$ core may influence the growth of $\mathrm{ZnS}$ and result in the formation of nanospines. The appropriate temperatures for the formation of nanospines were found to be $800-900{ }^{\circ} \mathrm{C}$. ZnS whiskers became coated with $\mathrm{BN}$ without the formation of nanospines at lower temperatures, while the ZnS nanoarchitectures were evaporated at higher temperatures.

It is an interesting and important problem to

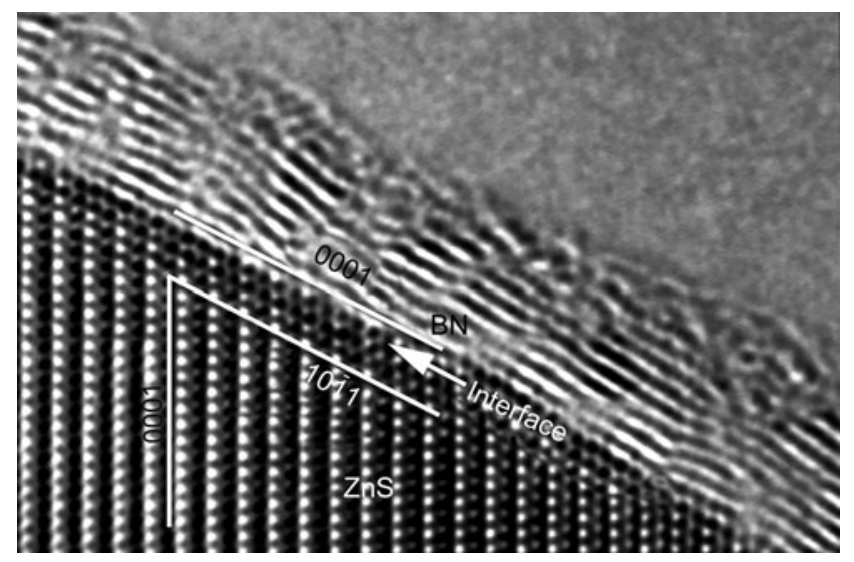

Figure 6 The interface between $\mathrm{BN}$ and $\mathrm{ZnS}$ showing periodic lattice connections between the (0001) facets of $B N$ and the (10 $\overline{1} 1)$ facets of $\mathrm{ZnS}$ 
design and prepare semiconductor nanomaterials with special structures. Crystals have intrinsic structural characteristics which are closely related to their growth habits. Consequently, the design and fabrication of nanostructures based on the crystallographic characteristics of materials should be a viable route to such materials. The above results show that each tricrystal of the tricrystal whiskers has a (1013) twin plane, which is a low-energy twin plane with atomic-scale twinning as shown in Fig. 4; such twin planes show good stability during the subsequent heat treatment as shown in Fig. 5. ZnS nanospine arrays were epitaxially grown in three [0001] directions on the three parts of the tricrystal, which is in accordance with the crystallographic characteristics of wurtzite. The twin boundary without the atomic-scale twinning exhibits poor stability during the subsequent heat treatment, as shown in Fig. 2(b).

\section{Conclusions}

ZnS tricrystal whiskers with $\{1013\}$ twin planes have been synthesized by thermal evaporationdeposition methods; ZnS nanospine arrays oriented in three [0001] directions were epitaxially grown on the three parts of the tricrystal. The results show that nanostructures can be designed and predicted by analyzing their crystallographic characteristics and that ordered and complex oriented nanostructures can be fabricated via step-by-step crystallographically-controlled chemical processes.

\section{Acknowledgements}

Y. Z. acknowledges financial support from the National Natural Science Foundation of China (20571082, 50772125), and the Science and Technology Commission of Shanghai (08JC1420700).

\section{References}

[1] Milliron, D. J.; Hughes, S. M.; Cui, Y.; Manna, L.; Li, J.; Wang, L. W.; Alivisatos, A. P. Colloidal nanocrystal heterostructures with linear and branched topology. Nature 2004, 430, 190-195.
[2] Lee, K. B.; Park, S.; Mirkin, C. A. Multicomponent magnetic nanorods for biomolecular separations. Angew. Chem. Int. Ed. 2004, 43, 3048-3050.

[3] Polyakov, B.; Daly, B.; Prikulis, J.; Lisauskas, V.; Vengalis, B.; Morris, M. A.; Holmes, J. D.; Erts, D. High-density arrays of germanium nanowire photoresistors. Adv. Mater. 2006, 18, 1812-1816.

[4] Hayden, O.; Agarwal, R.; Lieber, C. M. Nanoscale avalanche photodiodes for highly sensitive and spatially resolved photon detection. Nat. Mater. 2006, 5, 352356.

[5] Verheijen, M. A.; Immink, G.; de Smet, T.; Borgstrom, M. T.; Bakkers, E. P. A. M. Growth kinetics of heterostructured GaP-GaAs nanowires. J. Am. Chem. Soc. 2006, 128, 1353-1359.

[6] Zhu, Y.C.; Fujiwara, M. Installing dynamic molecular photomechanics in mesopores: A multifunctional controlled-release nanosystem. Angew. Chem.Int. Ed. 2007, 46, 2241-2244.

[7] Haas, I.; Gedanken, A. Synthesis of metallic magnesium nanoparticles by sonoelectrochemistry. Chem. Comm. 2008, 1795-1797.

[8] Hong, S.; Mirkin, C. A. A nanoplotter with both parallel and serial writing capabilities. Science 2000, 288, 18081811.

[9] Loo, Y.L.; Willett, R.; Baldwin, K. W.; Rogers, J. A. Interfacial chemistries for nanoscale transfer printing. J. Am. Chem. Soc. 2002, 124, 7654-7655.

[10] Wang, Z. L.; Kong, X. Y.; Zuo, J. M. Induced growth of asymmetric nanocantilever arrays on polar surfaces. Phys. Rev. Lett. 2003, 91,185502.

[11] Yan, H. Q.; He, R. G.; Johnson, J.; Law, M.; Saykally, R. J.; Yang, P. D. Dendritic nanowire ultraviolet laser array. J. Am. Chem. Soc. 2003, 125, 4728-4729.

[12]Manna, L.; Milliron, D. J.; Meisel, A.; Scher, E. C.; Alivisatos, A. P. Controlled growth of tetrapod-branched inorganic nanocrystals. Nat. Mater. 2003, 2, 382-385.

[13] Zhu, Y. C.; Bando, Y.; Xue, D. F.; Golberg, D. Oriented assemblies of ZnS one-dimentional nanostructures. Adv. Mater. 2004, 16, 831-834.

[14] Zhu, Y. C.; Bando, Y.; Xue, D.F.; Golberg, D. Nanocablealigned ZnS tetrapod nanocrystals. J. Am. Chem. Soc. 2003, 125, 16196-16197.

[15] Biswas, S.; Ghoshal T.; Kar S.; Chakrabarti S.; Chaudhuri S. ZnS nanowire arrays: Synthesis, optical and field emission properties. Cryst. Growth Des. 2008, 8, 2171-2176. 
[16] Biswas S.; Kar S.; Chaudhuri S.; Nambissan, P. M. G. Positron annihilation studies of defects and interfaces in ZnS nanostructures of different crystalline and morphological features. J. Chem. Phys. 2006, 125, 164719.

[17] Xiong, Q.; Chen, G.; Acord, J. D.; Liu, X.; Zengel, J. J.; Gutierrez, H. R.; Redwing, J. M.; Voon, L. C. L. Y.; Lassen, B.; Eklund, P. C. Optical properies of rectangular cross-sectional ZnS nanowires. Nano Lett. 2004, 4, 1663 $-1668$.

[18] Yamamoto, T.; Kishimoto, S.; lida, S. Control of valence states for ZnS by triple-codoping method. Physica $B$ 2001, 308, 916-919.

[19] Prevenslik, T. V. Acoustoluminescence and sonoluminescence. J. Lumin. 2000, 87-89, 1210-1212.

[20]Falcony, C.; Garcia, M.; Ortiz, A.; Alonso, J.C. Luminescent properties of ZnS: Mn films deposited by spray pyrolysis. J. Appl. Phys. 1992, 72,1525-1527.

[21] Fan, X.; Meng, X. M.; Zhang, X. H.; Shi, W. S.; Zhang, W. J.; Zapien, J. A.; Lee, C. S.; Lee, S. T. Dart-shaped tricrystal ZnS nanoribbons. Angew. Chem. Int. Ed. 2006, 45, 2568-2571.

[22] Ma, C.; Moore, D.; Li, J.; Wang, Z. L. Nanobelts, nanocombs and nanowindmills of wurtzite ZnS. Adv. Mater. 2003, 15, 228-231.

[23] Gautam, U. K.; Fang, X. S.; Bando, Y.; Zhan, J. H.; Golberg, D. Synthesis, structure, and multiply enhanced field-emission properties of branched ZnS nanotube-in nanowire core-shell heterostructures. ACS Nano 2008, 2, 1015-1021.

[24] Zhu, Y. C.; Bando, Y.; Xue, D. F. Spontaneous growth and luminescence of zinc sulfide nanotubes. Appl. Phys. Lett. 2003, 82, 1769-1771.

[25]Zhu, Y. C.; Bando, Y. ZnS-Zn nanocables and ZnS nanotubes. Chem. Comm. 2003, 836-837.
[26]Li, Q.; Wang, C. R. Fabrication of Zn/ZnS nanocable heterostructures by thermal reduction/sulfidation. Appl. Phys. Lett. 2003, 82, 1398-1370.

[27] Zhu, Y.C.; Bando, Y. Preparation and photoluminescence of single-crystal zinc selenide nanowires. Chem. Phys. Lett. 2003, 377, 367-370.

[28] Rana, R. K.; Zhang, L. Z.; Yu, J. C.; Mastai, Y.; Gedanken, A. Mesoporous structures from supramolecular assembly of in situ generated ZnS nanoparticles. Langmuir 2003, 19, 5904-5911.

[29] Yu, S. H.; Yoshimura, M. Shape and phase control of ZnS nanocrystals: template fabrication of wurtzite ZnS singlecrystal nanosheets and $\mathrm{ZnO}$ flake-like dendrites from a lamellar molecular precursor $\mathrm{ZnS} \cdot\left(\mathrm{NH}_{2} \mathrm{CH}_{2} \mathrm{CH}_{2} \mathrm{NH}_{2}\right)_{0.5}$. Adv. Mater. 2002, 14, 296-300.

[30] Xiong, S. I.; Xi, B. J.; Wang, C. M.; Xu, D.; Feng, X. M.; Zhu, Z. C.; Qian, Y. T. Tunable synthesis of various wurtzite $\mathrm{ZnS}$ architectural structures and their photocatalytic properties. Adv. Funct. Mater. 2007, 17, 2728-2738.

[31] Jiang, Y.; Meng, X. M.; Liu, J.; Xie, Z. Y.; Lee, C. S.; Lee, S. T. Hydrogen-assisted thermal evaporation synthesis of ZnS nanoribbons on a large scale. Adv. Mater. 2003, 15, 323-326.

[32] Zhu, Y. C.; Bando, Y.; Xue, D. F.; Xu, F. F.; Golberg, D. Insulation tubular $B N$ sheating on semiconducting nanowires. J. Am. Chem. Soc. 2003, 125, 14226-14227.

[33] Zhu, Y. C.; Bando, Y.; Yin, L. W. Design and fabrication of BN-sheathed ZnS nanoarchitectures. Adv. Mater. 2004, 16, 331-334.

[34] Brown I. D.; Altermatt, D. Bond-valence parameters obtained from a systematic analysis of the inorganic crystal structure database. Acta Crystallogr. B 1985, 41, 244-247. 